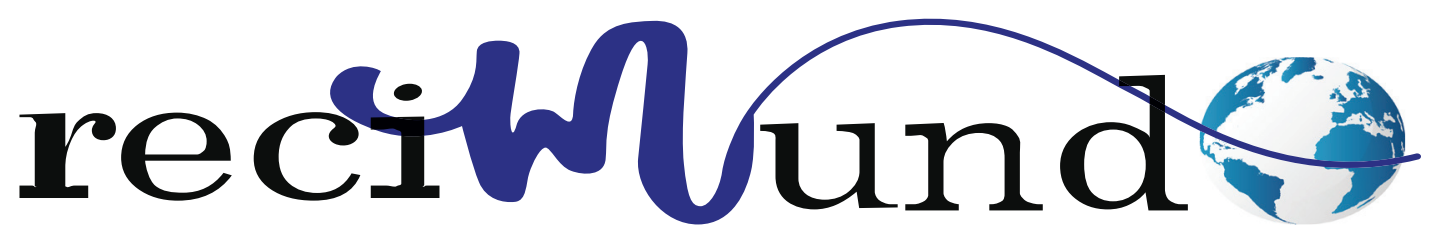

Revista Científica Mundo de la Investigación y el Conocimiento

DOI: 10.26820/recimundo/4.(1).esp.marzo.2020.210-218

URL: http://recimundo.com/index.php/es/article/view/796

EDITORIAL: Saberes del Conocimiento

REVISTA: RECIMUNDO

ISSN: 2588-073X

TIPO DE INVESTIGACióN: Artículo de Revisión

CÓDIGO UNESCO: 3205 Medicina Interna

PAGINAS: $210-218$

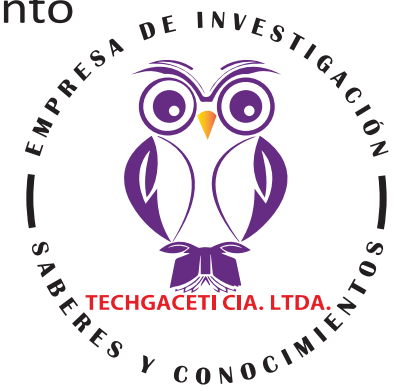

\title{
Osteomielitis aguda: manifestaciones clínicas, diagnóstico y tratamiento
}

Acute osteomyelitis: clinical manifestations, diagnosis and treatment

Osteomielite aguda: manifestações clínicas, diagnóstico e tratamento

\section{Eugenia Mariuxi Pincay Coello'; Ivanna Margarita Avilés Lúa2; Diego Mauricio Cabrera Moyano3;}

Cárdenas Rodríguez Jorge Daniel ${ }^{4}$

RECIBIDO: 20/11/2019 ACEPTADO: 29/01/2020 PUBLICADO: 05/03/2020

1. Médico; Investigadora Independiente; Guayaquil, Ecuador; epincayczs5@gmail.com; (D) https://orcid. org/0000-0002-7333-5149

2. Médico; Investigadora Independiente; Guayaquil, Ecuador; Ivannalua@outlook.es; (D) https://orcid.org/00000002-9053-9303

3. Médico; Investigador Independiente; Guayaquil, Ecuador; cabreramdiego@gmail.com; (D) https://orcid.org/0000-0001-7826-192X

4. Médico; Investigador Independiente; Guayaquil, Ecuador; docardenas3@gmail.com; (DD https://orcid.org/00000002-9728-9642

CORRESPONDENCIA

Eugenia Mariuxi Pincay Coello

epincayczs5@gmail.com

Guayaquil, Ecuador 


\title{
RESUMEN
}

La Osteomielitis aguda es una enfermedad que principalmente afecta a los niños, y en las zonas bajas de las piernas, sin embargo, no es difícil encontrar casos donde los afectados sean adultos. Hay casos donde se puede producir esta enfermedad en la boca, específicamente en la mandíbula. La Osteomielitis como tal tiene dos tipos de tratamiento, la antibioterapia y el quirúrgico, este último solo es utilizado cuando la enfermedad es muy grave o crónica. La metodología utilizada en la investigación, es de tipo revisión bibliográfica, apoyándose para ello en medios electrónicos para la obtención de la información. Entre las conclusiones es que esta enfermedad suele ser muy agresiva y puede generar mucho dolor, su proceso de diagnóstico es muy variado y va desde un simple examen de laboratorio hasta medicina nuclear, al ser detectada a tiempo el proceso de sanación con el tratamiento adecuado es total.

Palabras clave: Antibioticoterapia, Quirúrgico, Pierna, Tomografía, Etiología

\begin{abstract}
Acute osteomyelitis is a disease that mainly affects children, and in the lower areas of the legs, however, it is not difficult to find cases where those affected are adults. There are cases where this disease can occur in the mouth, specifically in the jaw. Osteomyelitis as such has two types of treatment, antibiotics and surgery, the latter is only used when the disease is very serious or chronic. The methodology used in the research is of the bibliographic review type, based on electronic means for obtaining the information. Among the conclusions is that this disease is usually very aggressive and can generate a lot of pain, its diagnostic process is very varied and ranges from a simple laboratory test to nuclear medicine, to be detected in time the healing process with the appropriate treatment is total.
\end{abstract}

Keywords: Antibiotic therapy, Surgical, Leg, Tomography, Etiology

\section{RESUMO}

A osteomielite aguda é uma doença que afeta principalmente crianças, e nas áreas inferiores das pernas, no entanto, não é difícil encontrar casos em que os afetados são adultos. Há casos em que esta doença pode ocorrer na boca, especificamente na mandíbula. Osteomielite, como tal, tem dois tipos de tratamento, antibióticos e cirurgia, o último só é usado quando a doença é muito grave ou crônica. A metodologia utilizada na pesquisa é do tipo revisão bibliográfica, baseada em meios eletrônicos para obtenção das informações. Entre as conclusões está a de que essa doença geralmente é muito agressiva e pode gerar muita dor, seu processo diagnóstico é muito variado e varia de um simples teste laboratorial à medicina nuclear, para ser detectado a tempo de que o processo de cicatrização com o tratamento adequado seja total.

Palavras-chave: Antibioticoterapia, Cirúrgica, Perna, Tomografia, Etiologia. 


\section{Introducción}

La osteomielitis es una enfermedad infecciosa que afecta a la porción medular y cortical del hueso, y generalmente está producida por la invasión de bacterias a través de una diseminación hematógena de tejidos adyacentes infectados, o por exposición de un hueso fracturado al exterior. Esta enfermedad se caracteriza por la presencia de una o más lesiones abscedadas que se expanden al tejido subperióstico, con necrosis y reabsorción del tejido óseo, dejando zonas integras de cortical, denominadas "secuestros" (Mollinedo Patzi, Morales Alba, Lanchipa Cruz, \& Aruquipa Salcedo, 2013, pág. 1761).

La clasificación se puede hacer según un gran número de criterios (etiología, patogenia, localización, evolución, características del huésped.), pero las más utilizadas son la patogénica de Waldvogel, que considera tres clases: hematógena, por contigüidad y asociada a insuficiencia vascular; y la de Cierny y Mader que establece doce grupos combinando la localización anatómica y la situación del huésped, y cuyo propósito es estandarizar el tratamiento (Riu, Pérez Quirós, Rodríguez Romero, \& Raya, 2009, pág. 30).

\section{Clasificación de Waldvogel (OMA)}

Hematógena:

- Propia de niños y adolescentes, se localiza en huesos largos metáfisis.

- En el adulto acostumbra a ser a nivel vertebral y en huesos largos a nivel de las diafisis.

Secundaria a un foco o inoculación directa:

- Fracturas abiertas, punciones cirugía.

- Propia de adultos pudiendo evolucionar hacia la cronicidad.

- La etiología suele ser polimicrobiana

Asociada a insuficiencia vascular y neuro- patía:

- Adultos.

- Antecedentes de diabetes e insuficiencia vascular.

- Afecta a los huesos del pie (pequeños traumatismos).

- Etiología suele ser polimicrobiana (Riu, Pérez Quirós, Rodríguez Romero, \& Raya, 2009, págs. 30-31).

Clasificación Cierny-Mader, según el estado anatómico

Medular: Hematógena.

- Superficial: Secundaria a un foco.

- Localizada: Secuestro de la cortical.

- Difusa: Afección ósea difusa.

- Según el estado fisiológico

- Huésped normal.

- Enfermedad de base sistémica o un compromiso local generalmente vascular.

- El tratamiento habitual no provoca mejora, después es necesario aplicar tratamiento antibiótico supresivo o amputación (Riu, Pérez Quirós, Rodríguez Romero, \& Raya, 2009, pág. 31).

- Etiología

- Recién nacidos: staphylococcus aureus, bacilos gramnegativos y estreptococos del grupo B.

- Mayores de 4 años: staphylococcus aureus, estreptococos del grupo A y coliformes.

- Adultos: staphylococcus aureus (Rojas Solano \& Vargas Rodríguez, 2018, págs. 55-56). 


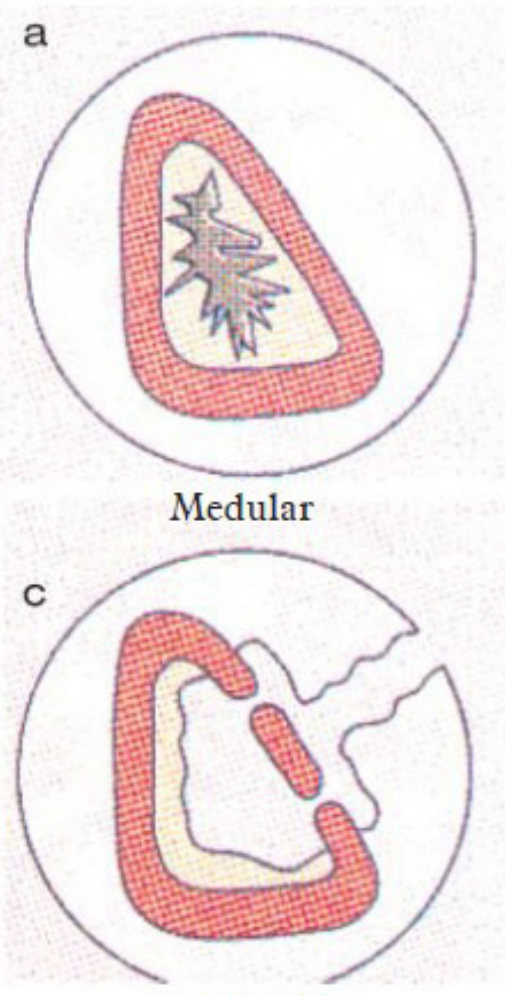

Localizada
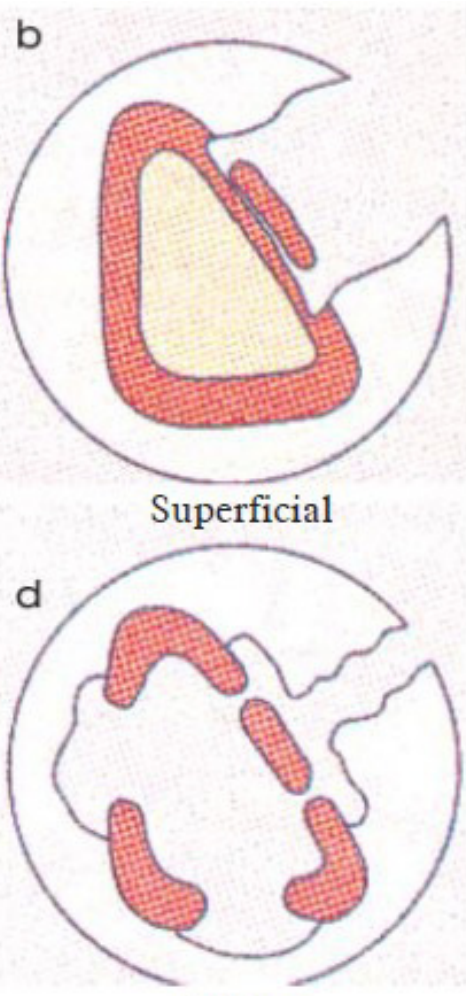

Difusa

Grafico 1. Tipos de Osteomielitis

Fuente: (Riu, Pérez Quirós, Rodríguez Romero, \& Raya, 2009)

Una de las primeras referencias que se tienen sobre esta entidad parece ser la que se le adjudica a Sir Benjamín Brodie (17831862), médico inglés, que describió por primera vez lo que sería conocido hasta nuestros días como absceso de Brodie, una de las formas crónicas de la osteomielitis, en un artículo llamado "Resultado de algunos casos crónicos de abscesos de la tibia" (Morán López \& Cueto Salas, 2001).

La localización anatómica de la osteomielitis hematógena depende de la edad. Desde el nacimiento hasta la pubertad predomina en los huesos largos de las extremidades. Las OM hematógenas, que representan el $17 \%$, se dan casi exclusivamente en la edad infantil y de estas, las localizadas en el maxilar, solo aparecen en edades tempranas de la vida (neonatos y lactantes) y se conocen en la literatura pediátrica con el nombre de "osteítis del germen dentario. "En los adultos suele localizarse en la columna verte- bral, puesto que las vértebras se vascularizan más que otros tejidos esqueléticos con la madurez. Cerca del $70 \%$ de las fracturas compuestas no se contaminan, cuando se realiza desbridamiento y se indica antibioticoterapia, de manera que solo del 2 al $9 \%$ se infectan. La OM de localización maxilar es poco frecuente si se compara con la que se manifiesta en las metáfisis de los huesos largos (del 1 al $2 \%$ ); también es baja su incidencia, teniendo en cuenta lo numerosas que son las infecciones dentarias y las lesiones traumáticas.10 Se presenta en cualquier edad y sin predominio sexual, aunque Goaz plantea que el mayor número de afectados corresponde al sexo femenino (Morán López \& Cueto Salas, 2001, pág. 54).

El retraso en el diagnóstico puede llevar a secuelas importantes, como: pérdida en la funcionalidad, destrucción ósea, formación de secuestros óseos, infección crónica localizada o afectación sistémica en huéspedes inmunodeficientes, por lo que es nece-

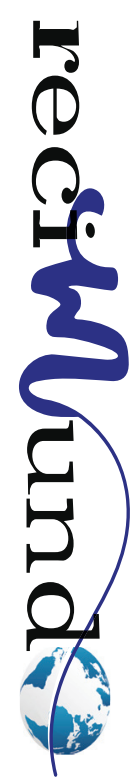


sario realizar el diagnóstico temprano ante un cuadro clínico sugestivo e implementar la terapia antibiótica adecuada (Félix, y otros, 2008).

\section{Metodología}

La metodología utilizada para la elaboración del presente trabajo de investigación, es una revisión bibliográfica, para lo cual se utilizaron medios electrónicos disponibles para la selección de otras investigaciones que ya han abordado el tema de esta investigación con mucha amplitud.

Resultados

\section{Manifestaciones clínicas}

En el examen físico del paciente con osteomielitis se encuentra dolor a la palpación de la región afectada, edema, rubor, aumento del calor local, en algunos casos se encuentran signos de infección de tejidos blandos, secreción local, fistulas, datos de traumatismo previo; en los pacientes con diabetes mellitus, la presencia de úlceras en miembros inferiores está fuertemente relacionada con presencia de osteomielitis hasta en un $60 \%$ de los casos. El paciente puede asociar fiebre, síntomas constitucionales importantes, sin que se correlacione con la gravedad de la osteomielitis. Se debe valorar sensibilidad y sistema vascular periférico (Ugalde Ovares \& Morales Castro, 2014).

El cuadro clínico varía según el tipo de osteomielitis, siendo así que en la osteomielitis aguda se presentan signos y síntomas clínicos como el estado febril de hasta $40^{\circ} \mathrm{C}$, fatiga y pérdida de peso, aumento de volumen de la región eritema, hipersensibilidad focal especialmente cuando se mueve una articulación cercana. En un niño muy pequeño los síntomas se detectan cuando él se niega a mover sus extremidades o a caminar, y llora por tanto dolor (Mollinedo Patzi, Morales Alba, Lanchipa Cruz, \& Aruquipa Salcedo, 2013, pág. 1764).

\section{Diagnostico}

\begin{tabular}{|l|l|}
\hline Tipo de Diagnostico & Observación \\
\hline \multirow{1}{*}{ Entre los hallaz- } \\
gos de las pruebas \\
de laboratorio se \\
pueden encontrar \\
elevación en la ve- \\
locidad de Eritrose- \\
dimentación (VES). \\
La proteína C reac- \\
tiva se eleva en las \\
primeras 8 horas, al- \\
canza el valor máxi- \\
mo a los 2 días y se \\
normaliza a la sema- \\
na de haber iniciado \\
el tratamiento; es útil \\
para el seguimiento \\
y para diferenciar \\
formas complica- \\
das. \\
Estas dos pruebas \\
de laboratorio se \\
utilizan tanto para \\
diagnóstico como \\
para seguimiento al \\
tratamiento. \\
Otros exámenes de \\
que se utilizan, son \\
el conteo de glóbu- \\
los blancos, dado \\
que se encuentra \\
aumento en los leu- \\
cocitos hasta en el \\
$60 \%$ de los casos \\
y los hemocultivos \\
que son positivos \\
entre el 20 y el 50\% \\
de los casos de os- \\
teomielitis aguda. \\
El mejor criterio \\
diagnóstico para la \\
osteomielitis es un \\
cultivo positivo de la \\
biopsia de hueso, y \\
una histopatología \\
consistente con ne- \\
crosis. \\
\hline logía
\end{tabular}




\begin{tabular}{|c|c|}
\hline Radiografías simple & 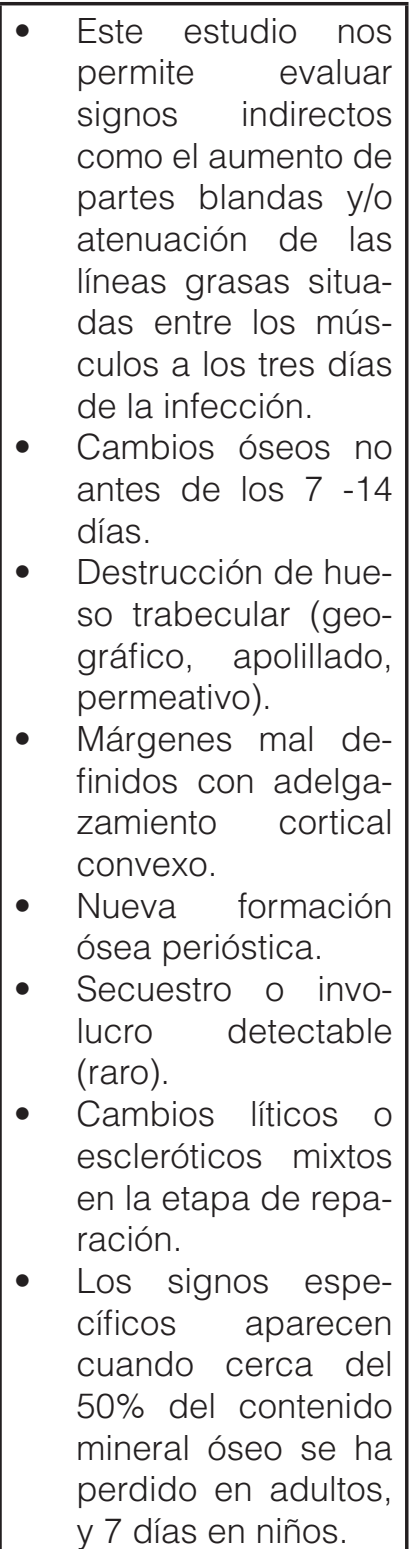 \\
\hline
\end{tabular}

\begin{tabular}{|c|c|}
\hline Ultrasonido & $\begin{array}{l}\text { En los infantes, la } \\
\text { fase temprana de la } \\
\text { osteomielitis es ini- } \\
\text { cialmente marcada } \\
\text { por la inflamación } \\
\text { edematosa de los } \\
\text { tejidos blandos más } \\
\text { profundos; ésta es } \\
\text { seguida más tarde } \\
\text { por un aspecto de } \\
\text { colección líquida } \\
\text { subperióstica fina, } \\
\text { de aproximadamen- } \\
\text { te } 2 \text { mm, y eco-libre } \\
\text { que eleva visible- } \\
\text { mente el periostio, } \\
\text { aunque éstos cam- } \\
\text { bios antes descritos } \\
\text { no son específicos. } \\
\text { En adultos el Ultra- } \\
\text { sonido solamente } \\
\text { puede detectar la } \\
\text { implicación del te- } \\
\text { jido blando asocia- } \\
\text { do. Puede detectar } \\
\text { características de la } \\
\text { osteomielitis a las } 48 \\
\text { horas del inicio de } \\
\text { la infección, mucho } \\
\text { antes de lo que lo } \\
\text { hacen las radiogra- } \\
\text { fías convencionales } \\
\text { (predominantemen- } \\
\text { te en los niños). } \\
\text { También es capaz } \\
\text { de localizar el sitio } \\
\text { y la extensión de la } \\
\text { infección, identificar } \\
\text { factores tales como } \\
\text { cuerpos extraños o } \\
\text { fístulas, y proporcio- } \\
\text { na una guía para la } \\
\text { aspiración o biop- } \\
\text { sia. }\end{array}$ \\
\hline
\end{tabular}


do afectado con el posterior reemplazo autólogo de hueso (Mollinedo Patzi, Morales Alba, Lanchipa Cruz, \& Aruquipa Salcedo, 2013).

El éxito terapéutico se relaciona con la precocidad en el diagnóstico, el tipo evolutivo (aguda o crónica), el agente causal, las características del foco de infección, la presencia de material de implante y el acierto en las medidas tomadas. El tratamiento de la osteomielitis comprende el uso de:

- Antimicrobianos.

- Cirugía.

- Rehabilitación física, psíquica y social.

- Tratamiento ortopédico (Riu, Pérez Quirós, Rodríguez Romero, \& Raya, 2009).

\section{Conclusiones}

La Osteomielitis puede tratarse de dos formas la quirúrgica y con antibioticoterapia. La quirúrgica que se emplea cuando existe osteomielitis crónica y la antibioticoterapia se emplea en los casos menos graves y para ella dependiendo de la clasificación de la enfermedad, se escoge el antibiótico o los antibióticos a administrar.

Cuando es detectada de una manera rápida y se inicia el proceso de tratamiento adecuado, se puede lograr una remisión total de la enfermedad. Los tratamientos quirúrgicos pueden durar meses en cambio con antibióticos entre 2 a 4 semanas se puede lograr la recuperación.

A pesar que esta enfermedad se presenta en una gran parte en niños, si existen casos donde se presenta en adultos, la enfermedad suele atacar principalmente de la rodilla para abajo pero existen casos estomatológicos y mandibulares.

Su diagnóstico suele ser por medio de palpación en la zona afectada y se pueden presentar síntomas de dolor, fiebre y otras sintomatologías. Se puede diagnosticar por medio de exámenes de laboratorio y microbiología, pero también existen otros métodos como: radiología, ultrasonido, tomografías y medicina nuclear.

\section{Bibliografía}

Félix, S. O., Del Prado, E., Masó, J., Kindelán, D., Romero, E., Guzmán, Z., \& García, L. (2008). Osteomielitis aguda en niños. Revista Información Científica, 60(4).

Mollinedo Patzi, M., Morales Alba, D., Lanchipa Cruz, R., \& Aruquipa Salcedo, C. (2013). Osteomielitis. Revista de Actualización Clínica Investiga, 34, 1761.

Morán López, E., \& Cueto Salas, A. (2001). Osteomielitis: Criterios actuales e importancia para el Estomatólogo. Revista Cubana de Estomatología, 38(1), 52-66.

Riu, L. M., Pérez Quirós, M., Rodríguez Romero, C., \& Raya, M. (2009). Tratamiento farmacológico de la osteomielitis. El Peu, 29(1), 30-38.

Rojas Solano, M. J., \& Vargas Rodríguez, P. (2018). Osteomielitis aguda características clínicas, radiológicas y de laboratorio. Revista Médica de Costa Rica y Centroamérica, 72(615), 347-354.

Ugalde Ovares, C. E., \& Morales Castro, D. (2014). Osteomielitis. Medicina Legal de Costa Rica, 31(1), 94-102. 
